\title{
A comparative study of the photocatalytic efficiency of metal oxide/hydroxyapatite nanocomposites in the degradation kinetic of ciprofloxacin in water
}

\author{
Jihane LABRAG ${ }^{l}$, Chaimaa EL BEKKALI ${ }^{1}$, Ikrame ES-SAIDI ${ }^{1}$, Habiba Bouyarmane ${ }^{1}$, Abdelaziz LAGHZIZIL ${ }^{1 *}$, \\ Mohamed KHAMAR $^{2}$, Didier ROBERT ${ }^{3}$ \\ ${ }^{1}$ Laboratory of Applied Chemistry of Materials, Faculty of Sciences, University of Mohammed V Rabat, Morocco \\ ${ }^{2}$ Civil Engineering and Environment Laboratory (LGCE), Higher School of Technology, SALE, Mohammed V University, \\ Rabat, Morocco \\ ${ }^{3}$ Institut de Chimie et Procédés pour l'Energie, l'Environnement et la Santé (ICPEES), University of Strasbourg, Saint- \\ Avold Antenna, Université de Lorraine, 12 rue Victor Demange, 57500, Saint-Avold, France.
}

\begin{abstract}
The photocatalytic efficiency of the metal oxide-hydroxyapatite photocatalysts prepared by soft chemistry using phosphate rock as calcium and phosphorus precursors has been investigated on the degradation kinetic of ciprofloxacin residues in water under UV-light (HPK125 W Lamp). The nature of metal oxide $\left(\mathrm{TiO}_{2}, \mathrm{ZnO}, \mathrm{Fe}_{2} \mathrm{O}_{3}\right)$, structure, surface area and pore-size distributions of the catalysts were analyzed by various techniques analyses. Association of nanoscale metal oxide with hydroxyapatite could enhance the sorption properties of the materials and confers them interesting photodegradation properties. The results of the kinetic study revealed that the activities of these photocatalysts were dependent on the oxide surface and the best activity was obtained with $\mathrm{TiO}_{2}$ /hydroxyapatite catalyst, which had the largest surface area. The effects of various operational parameters were thoroughly considered in order to achieve highest photodegradation efficiency. A correlation between the nature of associated metal oxide, surface properties, the sorption behavior and the photodegradation capacity of these composites could be establishedd
\end{abstract}

\section{Introduction}

Wastewater treatment is the world's first public health problem caused by the loading of various harmful substances from industrial, agricultural and domestic activities $[1,2]$. Assessing and managing risks from the presence of harmful chemicals in ecosystems are an approach that requires the integration of different environmental competencies. Despite the considerable efforts invested in the wastewater treatment, it is unfortunately still considered that currently a good amount of liquid waste is sent to the treatment plants, the rest being released into the natural environment. Faced with this worrying situation, the majority of research in the environmental sciences is moving towards the implementation of innovative processes for the treatment of these pollutants that are not or only slightly biodegradable [3]. The search for media able of recovering water remains one of the solutions for the treatment of water. There is a variety of methods for removing harmful substances from aqueous solutions. Among them, adsorption and photodegradation are considered the most widely used methods requiring effective support with good thermal stability, structural and surface properties [4, 5]. Several natural and synthetic materials have been evaluated [6-9]. Among them, apatite efficiency is better versus various pollutants by controlling their porosity and the chemical composition of their surfacee. Indeed, hydroxyapatite has been widely used thanks to its adsorption and ion exchange properties [6]. It is able to immobilize various heavy metals as well organic species. The development of new materials of high porosity makes it possible to have interesting physical properties. Its association with metal oxides such as titanium, zinc and iron inhibits novel properties of composites formed, particularly if apatite is prepared from natural phosphate as a low-cost precursor of calcium and phosphorus [7] Photodegradation on metal oxides such as $\mathrm{TiO}_{2}$ depends on the performance of the processes used and the nature of the photoactive as well as the operating conditions [8]. The influence of the specific surface of the support has been particularly studied. Nevertheless, other oxides $\left(\mathrm{ZnO}, \alpha-\mathrm{Fe}_{2} \mathrm{O}_{3}\right.$, etc.) prepared under suitable synthesis conditions lead to satisfactory photocatalysis results but require a modification of their surface by using a porous systems such as silica or clays which accelerate the adsorption rate and consequently their photodegradation $[9,10]$. For good adsorption, an affinity between the solid and the pollutant is first required such as performed is the case of grafted silica or modified titanium oxides [11]. For this purpose, the combination of metal oxide and hydroxyapatite may constitute a photoactive support for the degradation of organic residues in water $[8,10]$. Of these, the continuous release of drugs and their metabolites into the aquatic environment gives these molecules a character of pseudo-persistence. Their presence in aquatic environments depends on their physicochemical properties and in particular their solubility in water, the stability of molecules and their half-life [12]. The aim of this study was to compare the photocatalytic efficiency of three nanocomposites $\mathrm{ZnO}$ $\mathrm{HAp}, \mathrm{TiO}_{2}$ - $\mathrm{HAp}$ and $\mathrm{Fe}_{2} \mathrm{O}_{3}$ - $\mathrm{HAp}$ with $40 \%$ by weight percentage oxide:HAp on the basis of the previous results in relation to the chemical structure and the surface of the metal oxides.

\section{Experimental}

\subsection{Synthesis}

The pure hydroxyapatite powder (HAp) was prepared by a dissolution/reprecipitation method from a natural phosphate ore coming from the Bengurir region (Morocco) [7]. The dissolution of the ore was carried out

Corresponding author: laghzizi@fsr.ac.ma 
by introducing $20 \mathrm{~g}$ of this raw material in a $2 \mathrm{~L}$ reactor containing $500 \mathrm{ml}$ of bidistilled acidified water at $\mathrm{pH} 2$ by adding $20 \mathrm{ml}$ of concentrated $\mathrm{HNO}_{3}$ acid (65\%), with continuous stirring for $3 \mathrm{~h}$ at room temperature. After filtration, the recovered solution containing $\mathrm{Ca}^{2+}$ ions and $\mathrm{H}_{3} \mathrm{PO}_{4}$ were brought to $\mathrm{pH} 10$ by the addition of concentrated ammonia solution $(25 \%)$. The resulting precipitate was aged for $24 \mathrm{~h}$, filtered and washed with intermediate ultrasound irradiation steps to optimize grain dispersion. Finally, the powders are dried at $100^{\circ} \mathrm{C}$, and then heated at $500^{\circ} \mathrm{C}$ and $800^{\circ} \mathrm{C}$ for 3 hours.

The titanium, zinc and iron oxides were prepared by addition of concentrated ammonia $(25 \%)$ at $\mathrm{pH} 10$ to tetraisopropyl orthotitanate, zinc or iron nitrate, respectively. The gel-like materials were filtered, redispersed in deionized water under sonication and filtered again; this procedure being repeated three times. The procedure of the ultrasound irradiation can increase the stability of oxide phase affecting the cavitation phenomenon, enhances the exchange reactions occurring at the particle surface and limits the particle aggregation. The resulting gel-like materials were dried overnight at $100^{\circ} \mathrm{C}$ and calcined at $500^{\circ} \mathrm{C}$ and $800^{\circ} \mathrm{C}$ for $3 \mathrm{~h}$. For the preparation of the 40MHAp nanocomposites $(\mathrm{M}=\mathrm{Ti}, \mathrm{Zn}$, $\mathrm{Fe}$ ), the precursors of zinc, titanium and iron have been added to $200 \mathrm{ml}$ of the solution containing $\mathrm{Ca}^{2+}$ ions and phosphoric acid from addition of dissolved ore and a concentrated solution of $\mathrm{NH}_{4} \mathrm{OH}(25 \%)$ was added at $\mathrm{pH}=10$ with continuous stirring at room temperature. The theoretical weight percentage of the precursors oxide:HAp is chosen at $40 \%$ to have a better photoactivity, named 40ZnHAp, 40TiHAp and 40FeHAp. The resulting gelatinous precipitates were aged for 24 hours. After dispersion in water under ultrasonic irradiation, the precipitates were filtered and washed with water with intermediate steps of ultrasonic irradiation to optimize grain dispersion. Finally, the composite powders were dried in an oven at $100^{\circ} \mathrm{C}$ for 24 hours or at $500^{\circ} \mathrm{C}$ during 3 hours. The powders obtained are characterized by various analysis techniques, which are very useful for explaining the adsorption properties and the photodegrdation.

\subsection{Photodegradation tests}

Several reactors are used in photochemical studies, but the most used ones are those equipped with HPK lamps. Two types of filters are generally used according to experience. A quartz filter that lets all the wavelengths emitted by the light source pass at a high cost, a Pyrex filter that lets only certain wavelengths pass. In this work, we used a Pyrex reactor in which is placed a HPK high pressure mercury vapor lamp, which is contained in a Pyrex double-walled envelope allowing its cooling by circulating water. The protection of the reactor by the aluminum foil is necessary for the safety of the operator on the one hand and for good darkness on the other. The photochemical activity of photocatalysts heated at $500^{\circ} \mathrm{C}$ was evaluated under UV-light (HPK $125 \mathrm{~W}$ Lamp). A powder of $0.2 \mathrm{~g}$ was first dispersed in $100 \mathrm{~mL}$ of ciprofloxacin solution $\left(20 \mathrm{mg} \mathrm{L}^{-1}\right)$ and left to react in the dark for $30 \mathrm{~min}$. After, the solution was irradiated with
UV light under magnetic stirring. At different reaction times $(\mathrm{t})$, aliquots of the supernatant solution were taken with a $2 \mathrm{~mL}$ propylene syringe equipped with a $0.45 \mu \mathrm{m}$ filter and the equilibrium concentration of residual ciprofloxacin $\mathrm{q}_{\mathrm{t}}\left(\mathrm{mg} \mathrm{L}^{-1}\right)$ was measured by UV-visible spectroscopy and controlled by High Performance Liquid Chromatography (HPLC) for information on the by-products formed.

\section{Results and discussion}

\subsection{Characterization of the nanocomposite} powders

For the three nanocomposites, obtained 40ZnHAp, 40TiHAp, and 40FeHAp were characterized by XRD (Fig.1).
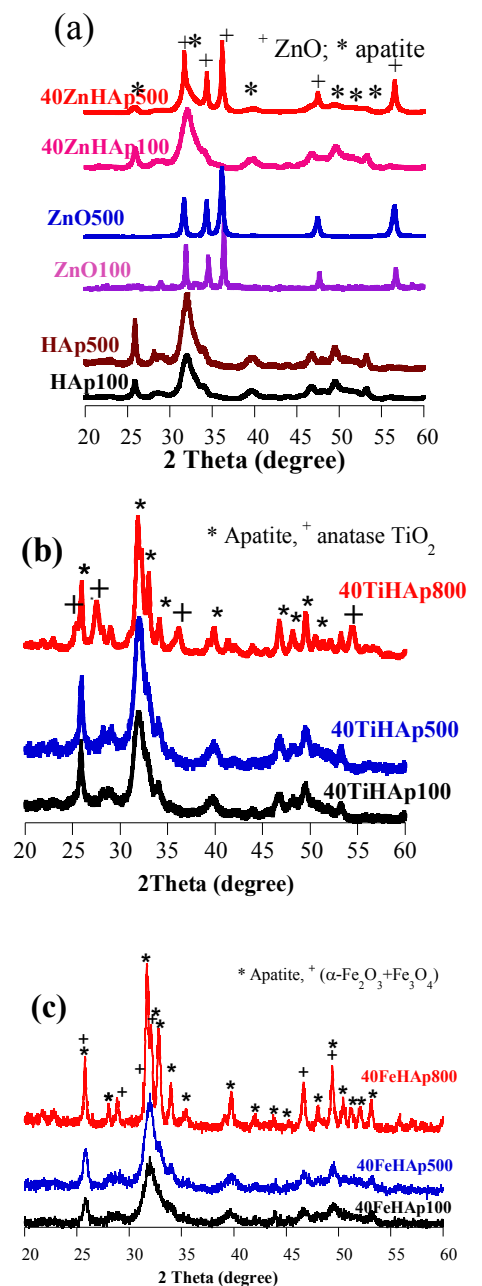

Fig. 1. XRD patterns of powders (a) 40ZnHAp (b) 40TiHAp and (c) 40FeHAp compared to those of pure HAp and metal oxides.

After drying at $100^{\circ} \mathrm{C}$, only broad diffraction peaks of apatite were identified and no oxide phase was detected. Moreover, after heat treatment at $500^{\circ} \mathrm{C}$, the diffraction peaks of the $\mathrm{ZnO}$ phase appear, but good crystallinity of the other oxides only appears at $800^{\circ} \mathrm{C}$. ICP-AES analyses were performed to determine the $\mathrm{Ca}, \mathrm{P}, \mathrm{Fe}, \mathrm{Ti}$ and $\mathrm{Zn}$ amounts in the nanocomposites (Table 1). Noting that the quantity of metals analyzed is close to that initially introduced. The specific surface area of the 
dried powders was calculated from $\mathrm{N}_{2}$ sorption/desorption measurements.

Table 1. Chemical analyses and surface characteristics of nanocomposites compared to individual metal oxide and HAP powders.

\begin{tabular}{lllllll}
\hline & $\mathrm{Ca} / \mathrm{P}$ & $\mathrm{Ti} / \mathrm{Zn} / \mathrm{Fe}$ & \multicolumn{2}{c}{$\mathrm{S}_{\mathrm{BET}}\left(\mathrm{m}^{2} / \mathrm{g}\right)$} & \multicolumn{2}{c}{$\mathrm{D}_{\mathrm{p}}(\mathrm{nm})$} \\
& & $(\%)$ & $100^{\circ} \mathrm{C}$ & $500^{\circ}$ & $100^{\circ} \mathrm{C}$ & $500^{\circ} \mathrm{C}$ \\
\hline 40TiHAp & 1.63 & 11.52 & 250 & 225 & $3.5 ;$ & $4.0 ;$ \\
& & & & & 9.0 & 9.0 \\
40ZnHAp & 1.65 & 15.61 & 250 & 155 & 11.0 & 11.0 \\
40FeHAp & 1.66 & 12.80 & 240 & 142 & $4.2 ;$ & $5.3 ;$ \\
& & & & & 9.0 & 9.2 \\
$\mathrm{ZnO}$ & - & - & 20 & 11 & 4.0 & 6.0 \\
$\mathrm{TiO}_{2}$ & - & - & 280 & 155 & 3.6 & 5.6 \\
$\mathrm{Fe}_{2} \mathrm{O}_{3}$ & - & - & 45 & 12 & 9.0 & 10.0 \\
$\mathrm{HAp}^{2}$ & 1.89 & - & 150 & 105 & 12.0 & 11.0 \\
\hline
\end{tabular}

From Table 1, 40TiHAp and 40ZnHAp surface area values are relatively higher than that of $40 \mathrm{FeHAp}$, and HAp. Therefore, the high specific surface value of 40TiHAp solid is due mainly to the contribution of $\mathrm{TiO}_{2}$ porosity, while that of 40ZnHAp is due to the inhibition of crystal growth of the particles obtained. In addition, the thermal treatment of the powders at $500^{\circ} \mathrm{C}$ tends to reduce the specific surface as described elsewhere [13]. We also determined the pore distribution, using the BJH method, of the composites, compared to those of HAp and metal oxides (Table 1). The combination of metal oxide with apatite leads to the improvement of the surface area of nanocomposites. These photoactive supports retain part of their porosity during heat treatment at $500^{\circ} \mathrm{C}$, which is very beneficial for the study of the adsorption coupled with the photodegradation of antibiotic under UV-light. TEM analysis of the nanocomposites shows well-defined aggregates in the form from 30 to $50 \mathrm{~nm}$, some of which contain dense nanoparticles of 5 to $20 \mathrm{~nm}$ (Fig. 2). After heat treatment at $500^{\circ} \mathrm{C}$ for 3 hours, coalescence of the hydroxyapatite particles was observed in agreement with the observed decrease in surface area values and the size of the dense nanoparticles appears to have increased. This suggests that the hydroxyapatite network limits the growth of the metal oxide nanoparticles in the composite powder.

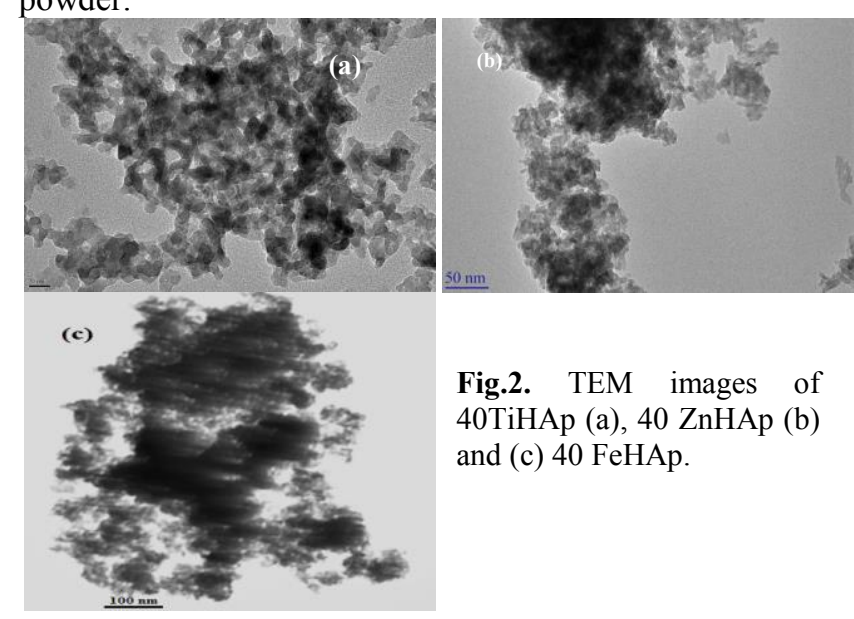

\subsection{Photodegradation process}

The kinetic study of the photocatalytic reactions of the ciprofloxacin on 40MHAp composites (M: Ti, Zn, Fe) calcined at $500^{\circ} \mathrm{C}$ was carried out with a concentration of $20 \mathrm{mg} \mathrm{L}^{-1}$ of the pollutant in the presence of $2 \mathrm{~g} \mathrm{~L}^{-1}$ of the catalyst under UV light. Before irradiating the solutions to be degraded, they are left in contact with the catalyst in the dark with continuous stirring for 30 minutes, the maximum time required for the ciprofloxacin adsorption by the photocatalyst. Under UV light, the results obtained from the photocatalytic degradation of the ciprofloxacin of $20 \mathrm{mg} \mathrm{L}^{-1}$ on different catalysts are shown in Fig.3. Without catalyst, no reduction in the initial concentration of ciprofloxacin as a function of time is observed, which reflects the absence of the photolysis reaction, while the presence of the catalyst contributes to the degradation of the majority of the studied antibiotic in water. However, the rate and percentage of degradation depends on the type of metal associated with hydroxyapatite. For 40TiHAp, the degradation efficiency is very good whereas that of 40ZnHAp is less pronounced and it becomes good in the case of the $40 \mathrm{FeHAp}$ composite.
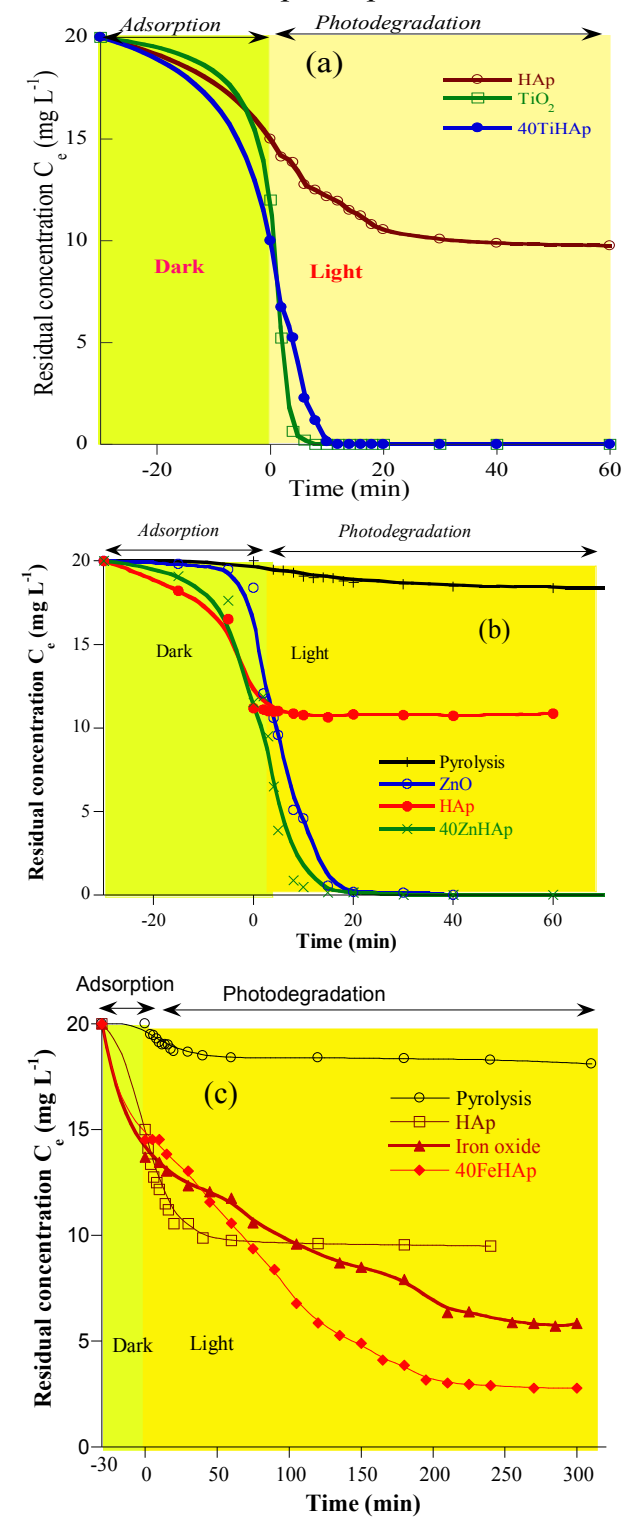

Fig.3 Kinetics of ciprofloxacin removal $\left(\mathrm{q}_{\mathrm{e}}\right.$ ) by (a) 40TiHAp, (b) 40ZnHAp and (c) 40FeHAp nanocomposite powders heated at $500^{\circ} \mathrm{C}$ compared to the individual pure $\mathrm{HAp}, \mathrm{TiO}_{2}$, 
$\mathrm{ZnO}$ and $\mathrm{Fe}_{2} \mathrm{O}_{3}$ oxides during a first 30 min period in the dark following by irradiation using a $125 \mathrm{~W}$ UV lamp.

The kinetic study of photodegradation of ciprofloxacin as a function of the initial CIP concentration from 10 to $30 \mathrm{mg} \mathrm{L}^{-1}$ was reported in Fig.4. With 5 ppm, the CIP is rapidly degraded because a smaller amount of molecules are supported or engaged by the catalyst and the degradation time is short (6 min). On the other hand, the abandonment of the CIP molecules near the catalyst hinders the photocatalytic reactions $(30 \mathrm{ppm})$ and the time is long $(15 \mathrm{~min})$. As a result, the more the solution is concentrated in CIP, the more time it takes to disappear.

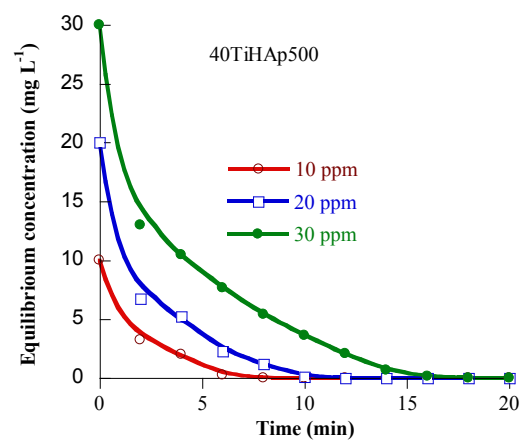

Fig.4. Initial concentration of CIP effect on its photodegradtion supported on $40 \mathrm{TiHAp}$ heated at $500^{\circ} \mathrm{C}$.

The absorbed photons by the 40MHAP composite catalysts activate the catalytic process in relation to the metal oxide associated to the porous HAp, and contribute to the formation of radicals in the composite. With a good photo-catalyst, the important transfer and accumulation of electrons towards the conduction band contributes to the formation of the $\mathrm{OH}^{\bullet}$ and $\mathrm{O}_{2}{ }^{--}$radicals, useful for triggering the degradation reactions of the antibiotic to release intermediates that can be fixed by the porous surface of the composite, confirmed by the absence of secondary peaks in the HPLC spectraevolving into $\mathrm{CO}_{2}, \mathrm{O}_{2}, \mathrm{H}_{2} \mathrm{O}, \mathrm{N}_{2} / \mathrm{NO}_{2}$ and $\mathrm{HF}$ at the end of the reaction (Fig.5).

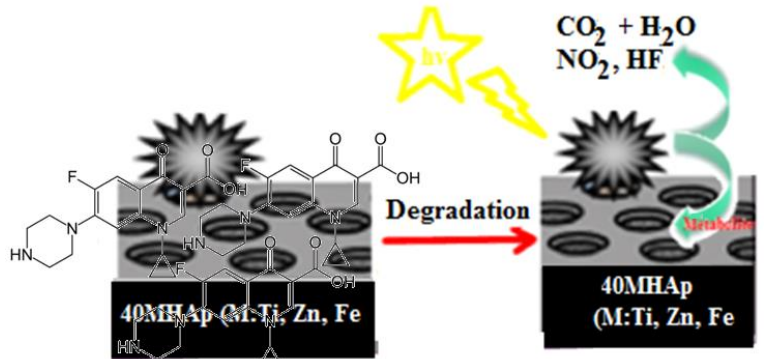

Fig.5 Proposed mechanisms of CIP photodegradation on porous nanocomposite powders.

The reuse of the catalysts was performed by their heat treatment at $500^{\circ} \mathrm{C}$ to maintain their photocatalytic activity. During photocatalytic degradation tests, the regenerated catalysts are reused for three cycles using 20 ppm as CIP with a dose $=2 \mathrm{~g} \mathrm{~L}^{-1}$ of catalyst. Results show that photocatalysts can be reused several times without losing their efficiency because they are thermally stable, one of the advantages of these nanocomposites and then this recycling process reduces the cost.

\section{References}

1. T. Heberer, K. Reddersen, A. Mechlinski, Water Science and Technology 46, 81 (2002)

2. A. Jelic, M. Gros, A. Ginebreda, R. CespedesSánchez, F. Ventura, M. Petrovic, D. Barcelo, Water Research 45, 1165 (2011).

3. T. Heberer, Toxicology Letters 131, 5 (2002).

4. M.M. Huber, S. Caninica, GY. Park, C.V Gunten, Environ. Sci. Technol. 37 , 1016 (2003).

5. N. Rioja , P Benguria , FJ Peñas, S Zorita, Environ. Sci. Pollut. Res. Int. 21, 11168 (2014).

6. S. Saoiabi, S. El Asri, A. Laghzizil, A. Saoiabi, J.L. Ackerman, T. Coradin, Chemical Engineering Journal 211-212, 233 (2012).

7. S. El Asri, A. Laghzizil, A. Saoiabi, A. Alaoui, K. El Abassi, R. M'Hamdi, T. Coradin, Colloid Surf. A 350, 73 (2009).

8. H. Bouyarmane, S. Saoiabi, I. El Hanbali, M. El Karbane, A. Rami,S. Masse, A. Laghzizil, T. Coradin, Eur. Phys. J. Special Topics 224, 1863 (2015).

9. C.B.D. Marien, M. Le Pivert, A. Azaïs, I. C. M'Bra, P. Drogui, A. Dirany, D. Robert, J. Hazard. Mater. 370, 164 (2019).

10. C. El Bekkali, H. Bouyarmane, M. El Karbane, S Masse, A. Saoiabi, T. Coradin, A. Laghzizil, Colloids and Surfaces A 539, 364 (2018).

11. D. Robert, V. Keller, N. Keller, Wiley-VCH, G. Weinheim (Eds.), Photocatalysis and Water Purification (2013) 145-178.

12. X. Zheng, S. Xu, Y. Wang, X. Suna, Y. Gao, B. Gao, J. Colloid and Interface Sci. 527, 202 (2018).

13. A. J. Nathanael, D. Mangalaraj, P.C. Chen, N. Ponpandian, Composites Science and Technology 70, 419 (2010). 